\title{
Neoadjuvant chemoradiotherapy followed by surgery in locally advanced squamous cell carcinoma of the vulva
}

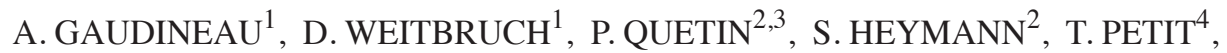 \\ P. VOLKMAR ${ }^{1}$, F. BODIN $^{1}$, M. VELTEN $^{5}$ and J.F. RODIER ${ }^{1}$ \\ Departments of ${ }^{1}$ Surgical Oncology and ${ }^{2}$ Radiotherapy, Regional Paul Strauss Cancer Centre, 67065 Strasbourg; \\ ${ }^{3}$ Department of Radiotherapy, Bon-Secours Hospital, 57000 Metz; Departments of ${ }^{4}$ Medical Oncology and ${ }^{5}$ Biostatistics, \\ Regional Paul Strauss Cancer Centre, 67065 Strasbourg, France
}

Received March 6, 2012; Accepted June 13, 2012

DOI: $10.3892 / \mathrm{ol} .2012 .831$

\begin{abstract}
Alternative therapies have been sought to alleviate mutilation and morbidity associated with surgery for vulvar neoplasms. Our prime objective was to assess tumor absence in pathological vulvar and nodal specimens following neoadjuvant chemoradiotherapy in locally advanced vulvar neoplasms. Data were retrospectively collected from January 2001 to May 2009 from 22 patients treated with neoadjuvant therapy for locally advanced squamous cell carcinoma of the vulva. Neoadjuvant treatment consisted of inguino-pelvic radiotherapy (50 Gy) in association with chemotherapy when possible. Surgery occurred at intervals of between 5 to 8 weeks. The median age of patients at diagnosis was 74.1 years. All patients were primarily treated with radiotherapy and 15 received a concomitant chemotherapy. Additionally, all patients underwent radical vulvectomy and bilateral inguino-femoral lymphadenectomy. Tumor absence in the vulvar and nodal pathological specimens was achieved for $6(27 \%)$ patients, while absence in the vulvar pathological specimens was only achieved for 10 (45.4\%) patients. Postoperative follow-up revealed breakdown of groin wounds, vulvar wounds and chronic lymphedema in $3(14.3 \%)$, $7(31.8 \%)$ and 14 cases $(63.6 \%)$, respectively. Within a median follow-up time of 2.3 years [interquartile range (IQR), 0.6-4.6], $12(54.6 \%)$ patients experienced complete remission and 6 cases succumbed to metastatic evolution within a median of 2.2 years (IQR, 0.6-4.6), with 1 case also experiencing perineal recurrence. Median survival time, estimated using the Kaplan-Meier method, was 5.1 years (IQR, 1.0-6.8). We suggest that neoadjuvant chemoradiotherapy may represent a reliable and promising strategy in locally advanced squamous cell carcinoma of the vulva.
\end{abstract}

Correspondence to: Professor Jean-François Rodier, Department of Surgical Oncology, Regional Paul Strauss Cancer Centre, 3 rue de la Porte de l'Hôpital, BP 42, 67065 Strasbourg, France E-mail: jrodier@strasbourg.unicancer.fr

Key words: vulvar neoplasms, squamous sell carcinoma, neoadjuvant chemoradiotherapy, surgery

\section{Introduction}

Vulvar neoplasms are an uncommon type of tumor accounting for only 3 to $5 \%$ of all gynecological cancers $(1,2)$; therefore, studies on the management of this type of cancer are scarce. At present, the standard treatments for vulvar neoplasms are radical vulvectomy and bilateral inguino-femoral lymphadenectomy. Studies of neoadjuvant radiotherapy aiming to shrink lesions that would have otherwise required pelvic exenteration, have suggested that radiotherapy may be used to reduce the extent of subsequent surgery or eradicate the disease in certain patients (2-4). Furthermore, concomitant radiotherapy and chemotherapy with 5-fluorouracil (5-FU) improved regression of various squamous cell carcinomas of the anus, esophagus, head, neck and cervix (3). This lead to the investigation of the use of induction chemoradiation as the primary therapy for squamous cell carcinoma of the vulva.

A number of retrospective analyses have established the feasibility of this new therapeutic approach, and suggested a therapeutic benefit of induction chemoradiation therapy for the treatment of vulvar neoplasms $(2,5-20)$. The majority of studies involved patients with locally advanced vulvar neoplasms, but certain studies also included recurrent tumor cases that were not eligible for surgical resection by radical vulvectomy. Most studies only considered patients with incomplete responses to chemoradiation therapy for limited resection of persistent vulvar disease. The majority of chemotherapy regimens consist of 5-FU in combination with either mitomycin C (MMC) or cisplatin (CDDP), and radiation doses have varied widely from 30 to 65 Gray (Gy). Despite the impressive response and local control rates, inhomogeneity of the study populations and wide variations in the radiation schemes invalidate attempts to compare results with those achieved from radiation therapy alone. To date, there have been no prospective randomized trials to assess the benefit of chemoradiation therapy in vulvar neoplasms. We reveal the results of a retrospective study on neoadjuvant therapy followed by radical surgery in a series of 22 patients with locally advanced squamous cell carcinoma of the vulva. The main objective was to estimate the rate of absence of the tumor in pathological vulvar and nodal specimens of this therapeutic approach. 


\section{Materials and methods}

Data were retrospectively collected from January 2001 to April 2010 from 22 patients treated with neoadjuvant therapy for primary locally advanced squamous cell carcinoma of the vulva at the Paul Strauss Cancer Centre (Strasbourg, France). The study was approved by the local ethics committee of the Paul Strauss Cancer Centre. Patient consent was obtained from the patient or the patient's family. Tumors were regarded as locally advanced if clinically estimated as T2 $>2.0 \mathrm{~cm}$ in diameter) with potential insufficient surgical margin (due to the proximity of vagina, urethra or anus) or T3 (involving the vagina, urethra or anus), according to the International Union Against Cancer (UICC) tumor-node-metastasis (TNM) classification. Induction treatment consisted of inguino-iliac-pelvic radiotherapy in 25 fractions of 50 Gy over 5 weeks using 4 isocentric beams. The beams were focalized using a multileaf collimator for all patients with a dose of $6 \mathrm{MV}$ anteriorly and $25 \mathrm{MV}$ posteriorly and laterally. When possible, treatment was combined, according to comorbid diseases, with radiosensibilization chemotherapy, using carboplatin (CBDCA) together with either 5 -FU (continuous perfusion of 5 -FU at $1000 \mathrm{mg} / \mathrm{m}^{2} /$ day and CBDCA with an area under the curve of 5 from day 1-4 of the first and fourth week of radiotherapy), paclitaxel (weekly perfusion of paclitaxel at $60 \mathrm{mg} / \mathrm{m}^{2}$ and CBDCA with an area under the curve of 2), or alone (weekly perfusion of CBDCA with an area under the curve of 2). The same members of the medical staff assessed the clinical objective responses throughout the treatment. All patients underwent radical vulvectomy and bilateral inguino-femoral lymphadenectomy as described by Morrow et al (21). Surgery was conducted at intervals of 5 to 8 weeks from neoadjuvant therapy. Follow-up clinical examinations were scheduled at 4-month intervals, inguino-pelvic CT scans were conducted twice a year and chest radiography once a year. The Kaplan-Meier method was used to estimate the median survival time.

\section{Results}

Tumor classification. Initial clinical tumor $(\mathrm{T})$ and nodal $(\mathrm{N})$ classifications of vulvar neoplasms were as follows: T2 $(n=7)$, T3 ( $n=15)$, N0 ( $n=10$; no lymph nodes involved), N1 ( $n=8$; lymph node metastases to 1 groin), $\mathrm{N} 2$ ( $\mathrm{n}=4$; lymph node metastases to both groins) (Table I). Squamous cell carcinomas of the vulva were primarily managed with surgery if clinically estimated to be $<\mathrm{T} 2$. Prior to treatment, all patients underwent a clinical examination of the pelvis and perineal lesions were biopsied. Systematic fine-needle aspiration cytology or microbiopsy of the inguinal nodes were not conducted. The median age of patients at diagnosis was 74.1 years [interquartile range (IQR), 50.7-80.9] and the median body mass index was $27.0 \mathrm{~kg} / \mathrm{m}^{2}$ (IQR, 23.0-30.0).

Radiotherapy and chemotherapy. All 22 patients completed the full course of neoadjuvant therapy; 22 patients received inguino-pelvic radiotherapy and $15(68.2 \%)$ received concomitant chemotherapy (2 had combined CBDCA and 5-FU, 11 had CBDCA alone and 2 had combined CBDCA and paclitaxel). The chemotherapy treatment induced moderate leucopenia in 2 patients. Radiotherapy was relatively well-tolerated and
Table I. Initial clinical classification of squamous cell carcinoma of the vulva according to the UICC TNM classification of malignant tumors.

\begin{tabular}{lcccc}
\hline & \multicolumn{3}{c}{$\mathrm{N}$} & \\
\cline { 2 - 4 } & 0 & 1 & 2 & Total \\
\hline $\mathrm{T}$ & & & & \\
$2(\%)$ & $5(71.4)$ & $2(28.6)$ & $0(00.0)$ & $7(31.8)$ \\
$3(\%)$ & $5(33.3)$ & $6(40.0)$ & $4(26.7)$ & $15(68.2)$ \\
Total & 10 & 8 & 4 & 22 \\
\hline
\end{tabular}

UICC, International Union Against Cancer; TNM, tumor-nodemetastasis.

the only acute toxic effect observed in 18 patients was vulvar inflammation, of which 5 cases were at grade 3 .

Radical vulvectomy and bilateral inguino-femoral lymphadenectomy. All 22 patients underwent radical vulvectomy and bilateral inguino-femoral lymphadenectomy with dissection of the superficial and deep inguinal lymph nodes without preservation of the external saphena vein. Surgery was conducted within a median time of 6.6 weeks (IQR, 5.0-8.0) following radiotherapy. The median number of lymph nodes removed per groin was 7 (IQR, 6-9).

Tumor absence. The absence of tumors in the vulvar and nodal pathological specimens was achieved for $6(27 \%)$ patients and absence in the vulvar pathological specimens only was achieved for $10(45.4 \%)$ patients. All but 1 patient had at least pathological partial response following neoadjuvant therapy. All 15 patients with T3 tumors were at least pT2 and 7 patients with T3 tumors were pT0. Inguinal lymph node involvement was bilateral in 2 patients and unilateral in 6. Inguinal lymph nodes were tumor-free in 3 of the $8 \mathrm{~N} 1$ patients and in all $4 \mathrm{~N} 2$ patients.

Follow-up. The median hospital stay for patients undergoing surgery was 18 days (IQR, 15-24). Postoperative follow-up revealed necrosis in 3 (14.3\%) cases, breakdown of groin wounds in $3(14.3 \%)$, breakdown of vulvar wounds in $7(31.8 \%)$, lymphocele in $14(63.6 \%)$ and chronic lymphedema in $14(63.6 \%)$.

A total of $6(27.3 \%)$ patients relapsed and succumbed to metastatic evolution (to the liver and lungs) within a median time of 2.2 years (IQR, 0.6-4.6). Additionally, 1 patient had a perineal recurrence treated by salvage posterior pelvic exenteration. Within a median follow-up time of 2.3 years (IQR, 0.6-4.6), 12 (54.6\%) patients were in complete remission. With the exception of the 6 recurrences, 4 patients succumbed to comorbid diseases. The median survival time was 5.1 years (IQR, 1.0-6.8).

\section{Discussion}

In this study, tumor absence in vulvar pathological specimens (pT0) was achieved in $45.4 \%$ of cases. Several retrospec- 
tive analyses have established the feasibility of neoadjuvant therapy in cases of vulvar neoplasms and suggested a therapeutic benefit (17-20,22-24). Lupi et al (17) treated 24 patients with combined 5-FU, MMC and irradiation divided into 2 courses of 36 and $18 \mathrm{~Gy}$. Although an objective response was observed in $22(91.6 \%)$ primary tumors, the vulvar pathological complete response rate was $8 / 22$ (36.3\%). Landoni et al (18) treated 41 patients with combined 5-FU, MMC and irradiation divided into 2 courses of 36 and $18 \mathrm{~Gy}$. Clinically and partially complete responses were observed in 34.7 and $53.1 \%$ of vulvar lesions, respectively, and a pathologically complete response of both vulvar and inguinal disease was detected in $31.0 \%$. Eifel et al (19) treated 12 patients with combined 5-FU, CDDP and irradiation to a total dose of $50 \mathrm{~Gy}$. Out of the 12 patients, $11(91.7 \%)$ demonstrated at least a partial response. Additionally, 4 out of the 8 patients who underwent vulvar resection had a pathologically complete response and remained disease-free for 1.4-3.1 years, and another clinically complete responder, who did not undergo surgery, was disease-free at 2.3 years. The most impressive response to neoadjuvant therapy is demonstrated in a Gynecology Oncology Group (GOG) phase II study (22). A total of 71 patients were treated with combined 5-FU, CDDP and irradiation to a dose of $47.6 \mathrm{~Gy}$. Clinically and pathologically complete tumor responses were observed in 48 and $70 \%$ of patients, respectively. Overall, only $2(3 \%)$ patients had unresectable disease following chemoradiation, and it was only necessary to conduct urinary and/or gastrointestinal diversion in 2 patients.

As this retrospective analysis evaluates the treatment efficacy in 22 patients treated over a 9 -year period, the chemotherapy protocol varied. In coherence with evidence-based medicine, CBDCA and 5-FU were used first. Vulvar inflammation following radiotherapy was deemed too intense, thus, the oncology team decided to switch to a chemotherapy protocol with CBDCA alone. Within a few years, the chemotherapy protocol was extended to CBDCA combined with paclitaxel due to a concomitant phase II study on advanced cervical cancer lead in the Paul Strauss Cancer Centre, using the same chemotherapy regimen (25). A total of 7 patients did not receive concomitant chemotherapy following geriatric evaluation due to heavy comorbidities.

In the present study, the reduction in tumor size occurred at the cost of important local morbidity represented by chronic lymphedema in $63.6 \%$ of cases. Few studies took this matter into account. Similar to the present study, the postoperative morbidity rate was high (65\%) in the study by Lupi et al (17), but lymphedema was observed in only 6 out of 22 patients. In order to potentially decrease local morbidity, our last patient benefited from delayed groin wound healing with vacuum-assisted closure (VAC). In this case, the duration of the follow-up was not long enough to indicate a possible control of local morbidity. Nevertheless, this technique appears to be of interest as it increases the rate of granulation tissue formation and reduces chronic edema, which leads to increased localized blood flow and neovascularization (26). Further studies to indicate the potential benefit in terms of local morbidity control are required.

We observed a recurrence rate of $27.3 \%$ within a median of 2.2 years. With a median follow-up time of 2.3 years, the complete remission rate was $54.6 \%$. Finally, median survival time was 5.1 years. In comparison, Lupi et al (17) demonstrated a recurrence rate of $31.8 \%$ following a median follow-up time of 2.8 years. Gerszten et al (20) treated 18 patients with combined 5-FU, CDDP and irradiation to a dose of $50 \mathrm{~Gy}$. There were $13(72.2 \%)$ complete responders of whom 12 remained disease-free at 2.1 years, and 5 (27.8\%) partial responders of whom 2 suffered local recurrences. Finally, within a median follow-up of 4.2 years, the local recurrence rate was $16 \%$ in the GOG phase II study (22).

In the present study, interpretation of lymph node response to neoadjuvant therapy remains difficult, due to the absence of initial anatomopathological proof of invasion. Only 2 N0 patients were pN1 and 1 was pN2. Other studies have established the therapeutic benefit of neoadjuvant therapy on lymph node metastasis. In the GOG phase II study (23), 40 patients with unresectable N2/N3 groin lymph nodes underwent neoadjuvant chemoradiation therapy. The nodes became resectable for $38(95 \%)$ of them. Pathological complete response was observed in $15(41 \%)$ patients. Overall, 20 patients were alive without evidence of recurrent cancer, and 5 succumbed without evidence of recurrence.

Neoadjuvant therapy may represent a reliable and promising therapeutic strategy for locally advanced squamous cell carcinoma of the vulva. It may improve operability and decrease the limitations of pelvic exenterative procedures to the rare progressing or non responding patients. Larger clinical trials are required to confirm long term data on overall survival and disease-free survival.

\section{References}

1. Rogers LJ, Howard B, Van Wijk L, et al: Chemoradiation in advanced vulval carcinoma. Int J Gynecol Cancer 19: 745-751, 2009.

2. Thomas GM, Dembo AJ, Bryson SCP, Osborne R and DePetrillo AD: Changing concepts in the management of vulvar cancer. Gynecol Oncol 42: 9-21, 1991.

3. Boronow RC, Hickman BT, Reagan MT, Smith RA and Steadham RE: Combined therapy as an alternative to exenteration for locally advanced vulvovaginal cancer. II. Results, complications and dosimetric and surgical considerations. Am J Clin Oncol 10: 171-181, 1987.

4. Hacker NF, Berek JS, Juillard GJF and Lagasse LD: Preoperative radiation therapy for locally advanced vulvar cancer. Cancer 54: 2056-2061, 1984.

5. Iversen T: Irradiation and bleomycin in the treatment of inoperable vulval carcinoma. Acta Obstet Gynecol Scand 61: 195-197, 1982.

6. Scheistroen M and Trope C: Combined bleomycin and irradiation in preoperative treatment of advanced squamous cell carcinoma of the vulva. Acta Oncol 32: 657-661, 1992.

7. Levin W, Goldberg G, Altaras M, Bloch B and Shelton MG: The use of concomitant chemotherapy and radiotherapy prior to surgery in advanced stage carcinoma of the vulva. Gynecol Oncol 25: 20-25, 1986.

8. Evans LS, Kersh CR, Constable WC and Taylor PT: Concomitant 5-fluorouracil, mitomycin-C, and radiotherapy for advanced gynecologic malignancies. Int J Radiat Oncol Biol Phys 15: 901-906, 1988 .

9. Wahlen SA, Slater JD, Wagner RJ, et al: Concurrent radiation therapy and chemotherapy in the treatment of primary squamous cell carcinoma of the vulva. Cancer 75: 2289-2294, 1995.

10. Akl A, Akl M, Boike G, Hebert J and Graham J: Preliminary results of chemoradiation as a primary treatment for vulvar carcinoma. Int J Radiat Oncol Biol Phys 48: 415-420, 2000.

11. Mulayim N, Foster Silver D, Schwartz PE and Higgins S: Chemoradiation with 5-fluorouracil and mitomycin $\mathrm{C}$ in the treatment of vulvar squamous cell carcinoma. Gynecol Oncol 93: 659-666, 2004. 
12. Koh WJ, Wallace HJ III, Greer BE, et al: Combined radiotherapy in the management of local-regionally advanced vulvar cancer. Int J Radiat Oncol Biol Phys 26: 809-816, 1993.

13. Han SC, Kim DH, Higgins SA, Carcangiu ML and Kacinski BM: Chemoradiation as primary or adjuvant treatment for locally advanced carcinoma of the vulva. Int J Radiat Oncol Biol Phys 47: 1235-1244, 2000.

14. Berek JS, Heaps JM, Fu YS, Julliard GJ and Hacker NF: Concurrent cisplatin and 5-fluorouracil chemotherapy and radiation therapy for advanced-stage squamous carcinoma of the vulva. Gynecol Oncol 42: 197-201, 1991.

15. Russell AH, Mesic JB, Scudder SA, et al: Synchronous radiation and cytotoxic chemotherapy for locally advanced or recurrent squamous cancer of the vulva. Gynecol Oncol 47: 14-20, 1992.

16. Cunningham MJ, Goyer RP, Gibbons SK, Kredentser DC, Malfetano JH and Keys H: Primary radiation, cisplatin, and 5 -fluorouracil for advanced squamous carcinoma of the vulva Gynecol Oncol 66: 258-261, 1997.

17. Lupi G, Raspagliesi F, Zucali R, et al: Combined preoperative chemoradiotherapy followed by radical surgery in locally advanced vulvar carcinoma: a pilot study. Cancer 77: 1472-1478, 1996.

18. Landoni F, Maneo A, Zanetta G, et al: Concurrent preoperative chemotherapy with 5-fluorouracil and mitomycin $\mathrm{C}$ and radiotherapy (FUMIR) followed by limited surgery in locally advanced and recurrent vulvar carcinoma. Gynecol Oncol 61: 321-327, 1996

19. Eifel PJ, Morris M, Burke TW, Levenback C and Gershenson DM: Prolonged continuous infusion cisplatin and 5-fluorouracil with radiation for locally advanced carcinoma of the vulva. Gynecol Oncol 59: 51-56, 1995.
20. Gerszten K, Selvaraj RN, Kelley J and Faul C: Preoperative chemoradiation for locally advanced carcinoma of the vulva. Gynecol Oncol 99: 640-644, 2005.

21. Morrow CP, Curtin JP and Lopez de la Osa E: Radical total vulvectomy. In: Gynecologic Cancer Surgery. Churchill Livingstone Inc., New York, pp414-432, 1996.

22. Moore DH, Thomas GM, Montana GS, Saxer A, Gallup DG and Olt G: Preoperative chemoradiation for advanced vulvar cancer: a phase II study of the Gynecologic Oncology Group. Int J Radiat Oncol Biol Phys 42: 79-85, 1998.

23. Montana GS, Thomas GM, Moore DH, et al: Preoperative chemo-radiation for carcinoma of the vulva with N2/N3 nodes: a gynecologic oncology group study. Int J Radiat Oncol Biol Phys 48: 1007-1013, 2000.

24. Tomao F, Di Tucci C, Marchetti C, Perniola G, Bellati F and Panici PB : Role of chemotherapy in the management of vulvar carcinoma. Crit Rev Oncol Hematol 82: 25-39.

25. Lhomme C, Petit T, Largillier R, Mayer F, Floquet A, Rey A, Jimenez $M$ and Haie-Meder C: Concomitant weekly carboplatin $(\mathrm{CB})$ and paclitaxel $(\mathrm{P})$ with pelvic radiotherapy $(\mathrm{RT})$ for the treatment of advanced cervical cancer (ACC): A FNCLCC gynecologic group phase I trial. J Clin Oncol: ASCO abs. 5542, 2007.

26. Argenta LC and Morykwas MJ: Vacuum-assisted closure: a new method for wound control and treatment: clinical experience. Ann Plast Surg 38: 563-576, 1997. 